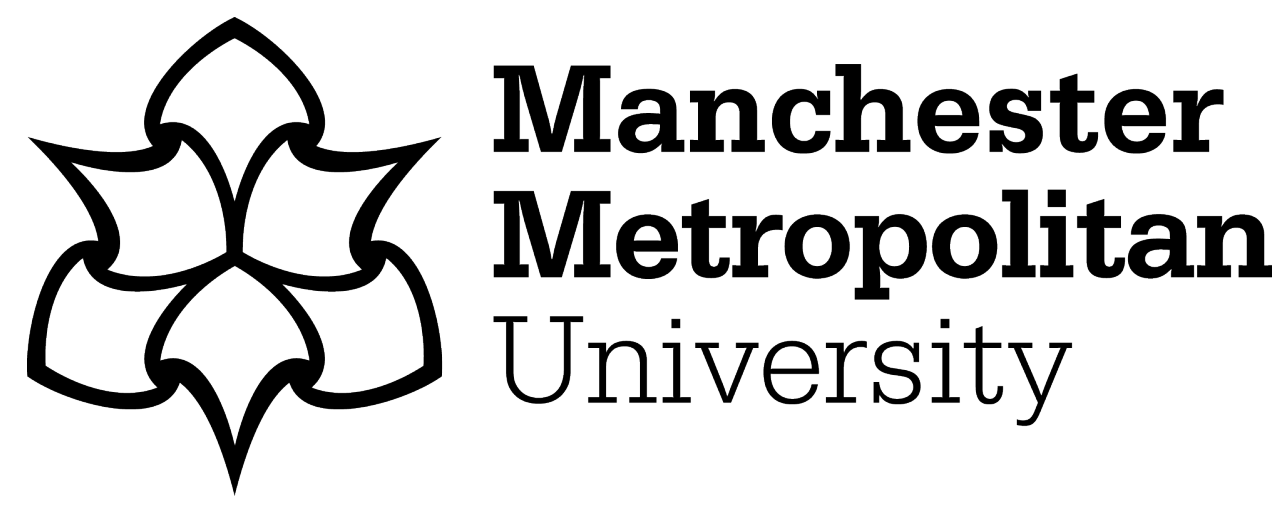

Hall, Elliott CR, Larruskain, Jon, Gil, Susana M, Lekue, Jose A, Baumert, Philipp, Rienzi, Edgardo, Moreno, Sacha, Tannure, Marcio, Murtagh, Conall F, Ade, Jack D, Squires, Paul, Orme, Patrick, Anderson, Liam, WhitworthTurner, Craig M, Morton, James P, Drust, Barry, Williams, Alun G and Erskine, Robert M (2020) An injury audit in high-level male youth soccer players from English, Spanish, Uruguayan and Brazilian academies. Physical Therapy in Sport, 44. pp. 53-60. ISSN 1466-853X

Downloaded from: https://e-space.mmu.ac.uk/625673/

Version: Accepted Version

Publisher: Elsevier

DOI: https://doi.org/10.1016/j.ptsp.2020.04.033

Usage rights: Creative Commons: Attribution-Noncommercial-No Derivative Works 4.0

Please cite the published version 


\section{AN INJURY AUDIT IN HIGH-LEVEL MALE YOUTH SOCCER PLAYERS FROM ENGLISH, SPANISH, URUGUAYAN AND BRAZILIAN ACADEMIES}

Elliott CR Hall ${ }^{1,10}$, Jon Larruskain ${ }^{2}$, Susana M Gil ${ }^{3}$, Jose A Lekue ${ }^{2}$, Philipp Baumert ${ }^{1}$, Edgardo Rienzi $^{4}$, Sacha Moreno ${ }^{4}$, Marcio Tannure ${ }^{5}$, Conall F Murtagh ${ }^{1,6}$, Jack D Ade ${ }^{1,6}$, Paul Squires ${ }^{6}$, Patrick Orme ${ }^{7}$, Liam Anderson ${ }^{1,8}$, Craig M Whitworth-Turner ${ }^{9}$, James P Morton ${ }^{1}$, Barry Drust ${ }^{1}$, Alun G Williams ${ }^{10,11}$ and Robert M Erskine ${ }^{1,11}$

${ }^{1}$ School of Sport and Exercise Sciences, Liverpool John Moores University, Liverpool, UK;

${ }^{2}$ Medical Services, Athletic Club, Lezama, Spain;

${ }^{3}$ Department of Genetics, Physical Anthropology and Animal Physiology, Faculty of Science and Technology,

University of the Basque Country (UPV/EHU), Leioa, Spain;

${ }^{4}$ Club Atlético Peñarol, Estadio Campeón del Siglo, Montevideo, Uruguay;

${ }^{5}$ Clube de Regatas do Flamengo, Rio de Janiero, Brazil;

${ }^{6}$ Liverpool Football Club, Liverpool, UK;

${ }^{7}$ Bristol City Football Club, Bristol, UK;

${ }^{8}$ Crewe Alexandra Football Club, Crewe, UK;

${ }^{9}$ Athletics Department, North Carolina State University, Raleigh, North Carolina, USA;

${ }^{10}$ Department of Sport and Exercise Sciences, Manchester Metropolitan University, Manchester, UK;

${ }^{11}$ Institute of Sport, Exercise and Health, University College London, London, UK.

\section{Address for correspondence:}

Dr Rob Erskine, PhD,

School of Sport and Exercise Sciences,

Liverpool John Moores University,

Liverpool, L3 3AF, United Kingdom

Email: R.M.Erskine@ ljmu.ac.uk

Tel: +44 1519046256

Fax: +44 1519046284

ORCID: 0000-0002-5705-0207

Twitter: @RMErskine

Key words: football; epidemiology; paediatric; injury risk; adolescence

\section{Acknowledgements:}

We are extremely grateful to the following individuals for their assistance with data collection: Sam Temple, Dr Mateo Gamarra, Dr Emiliano Vigna, Dr Gustavo Schmitner, Dr Luisina Passarello, Daniel Silva, Diego Morena, Bruno Jotta Costa and John Chaffe. 


\section{Highlights}

- Muscle injuries were the most common injury type in 624 youth soccer players

- The thigh was the most common injury location sustained in a single season

- Injury type and location were similar in players playing in different countries

- Players in the U14 and U16 age groups suffered relatively more severe injuries

- This suggests maturation affects injury risk in this under-researched population 


\section{$\underline{\text { Abstract }}$}

\section{Objectives}

To investigate the most common types and locations of injuries in high-level youth soccer players (YSP).

\section{Design}

Prospective cohort surveillance study.

\section{Setting}

Professional soccer club academies.

\section{Participants}

Six hundred twenty-four high-level YSP [Under 9 (U9) to U23 year-old age groups] from academies in England, Spain, Uruguay and Brazil.

\section{Main Outcome Measures}

The type, location and severity of injuries were recorded during one season. Injury severity was compared between age groups, with injury type and location compared between nations.

\section{Results}

Four hundred forty-three training or match injuries were recorded, giving an injury rate of 0.71 per player. Noncontact injuries were most common (58.5\%), with most (44.2\%) resolved between 8 and 28 days. Most injuries (75.4\%) occurred in the lower limbs, with muscle (29.6\%) the most commonly injured tissue. U14 and U16 suffered a greater number of severe injuries relative to U12 and U19/U20/U23/Reserves. Tendon injury rate was higher in Brazil vs. Spain $(p<0.05)$, with low back/sacrum/pelvis injury rate highest in Spain $(p<0.05)$.

\section{Conclusions}

The proportion of severe injuries in U14 and U16 suggests YSP injury risk is maturation-dependent. Minimal differences in type and location between high-level YSP from four different countries suggest injury rates in this population are geographically similar. 
The epidemiological study of sports injuries is imperative for injury prevention, by assisting in the identification of common injuries and their aetiology [1]. Accordingly, an injury audit provides stakeholders with evidence to enable them to advocate which factors likely influence injury occurrence and explore which may be modified to reduce injury risk [2]. An audit also forms the primary step of any injury prevention process [1], identifying which injuries occur, how often, and the extent of their impact upon a player or team. Subsequently, the understanding of injury occurrence is challenged and risk factors assumed to contribute toward, or cause injury, are proposed. Only after this step can the design and implementation of preventative strategies be considered in an attempt to reduce injuries. The cyclic process should then revisit the initial audit phase to evaluate the effectiveness of preventative measures on injury occurrence.

Identifying common types, circumstances and anatomical locations of soccer injuries highlights which have the greatest impact on player availability [3]. When many similar injuries are observed, it is logical that those injuries receive greatest attention compared to rare injuries affecting fewer players and teams. However, some infrequent injuries can be severe, causing the lengthiest absence to players, and may be career-threatening. Accordingly, the identification of severe yet less frequent injuries is also important, particularly as time lost to injury threatens the long-term development of youth players [4, 5]. In addition, player availability is closely linked to team success [6], meaning injury reduction is of significance to numerous stakeholders within the sport [7-9].

A considerable body of literature describes injury in soccer, with a large proportion derived from professional adult players. However, research on injury in youth soccer players (YSP) is also available. Whilst existing evidence guides researchers toward the most commonly cited types, causes and locations of injury, it is important to perform regular injury audits to ensure injury prevention strategies remain focussed on those posing the greatest problem. Furthermore, in populations where the number of injury audits are limited, the novel outcomes of new audits can assist in the study of risk factors specific to those populations.

The majority of injury-related absence in professional players and YSP is typically caused by soft-tissue injury $[4,10]$ and a large proportion of soccer injuries occur through non-contact situations [10-13]. Injuries primarily occur within the lower extremities [14], particularly in muscles such as those of the thigh [12, 15], with ligament injury also common [4]. In YSP, contusions, bruises and tendinopathies are also present [16]. With biological maturity occurring at different chronological ages [17], YSP in the same age categories often exhibit considerable anthropometric differences [18], which may impact their tolerance to training loads and their risk of injury. We aimed to audit the injuries suffered by high-level YSP over the course of one competitive season and 
hypothesised that the most frequently reported injury types would be muscle and ligament, and would primarily be non-contact. Furthermore, different coaching, playing and training styles may exist between countries and continents, which may influence the type and frequency of injuries suffered. However, despite some previous studies reporting YSP injury data from different nations [4, 16, 19], it is currently unknown if injuries in YSP differ when countries are directly compared with one another. Therefore, we also sought to investigate for the first time whether differences existed between high-level YSP from four different nations with respect to the most common injury types suffered across a single soccer season. We hypothesised that the lower limbs would incur the greatest proportion of injuries with minimal differences between nations, and that the thigh, knee and ankle would be among the most common locations. Finally, injuries reportedly peak in specific months of the season $[4,11]$ and we sought to investigate whether a similar pattern existed in our cohort.

\section{$\underline{\text { Materials and Methods }}$}

\section{Participants and study period}

The cohort included 624 high-level male YSP aged 9-23 years from the academies of eight professional soccer clubs from England, Spain, Uruguay and Brazil. Of the five English academies, two were categorised under the Premier League's Elite Player Performance Plan (EPPP) as Category 1 and two were Category 2. One English academy operated independently of the EPPP and competed regularly with Category 1 academies (Under 23 level). The Uruguayan academy was of the highest national category (Category A). There is no classification system for soccer academies in Spain or Brazil, however, the Spanish and Brazilian academies included in this audit are recognised as among the most successful in their respective countries. Participant characteristics are described in Table 1. The three youngest age groups were combined due to small numbers, and the U17 and U18 age groups were combined because no U17 age group exists in England under the Premier League's EPPP. The U19, U20, Reserves and U23 groups were combined, as only the U23 age group exists in England, and because player ages in the U19, U20 and Reserve teams of non-English clubs were similar to that of the English U23 teams. All players participated in regular soccer training and competition, which was in accordance with the Premier League's EPPP for the English clubs. Injuries were prospectively recorded during the 2011/12 to 2017/18 seasons. The number of seasons per club within this period ranged from one to seven, with only one season per player, per club included within the injury audit. The selected season corresponded to the season where the greatest number of players were available from each academy. This resulted in records for 223 players from the 2014/15 season (two clubs), 17 players from the 2016/17 season (one club) and 384 players from the 2017/18 season (five 
clubs). No player records contributed to more than one soccer season, in order to ensure equal comparison and reduce the influence of re-injuries. Written informed consent to participate in this audit was collected from club officials and players, with parental consent and player assent collected for all participants less than 16 years of age. The study received approval from Liverpool John Moores University Research Ethics Committee.

Table 1. Participant characteristics. Data are mean \pm SD.

\begin{tabular}{lcccc}
\hline Age Group & Number of players (\%) & Age (years) & Height $(\mathbf{m})$ & Body mass $(\mathbf{k g})$ \\
\hline U9, U10, U11 & $66(10.6)$ & $10.3 \pm 0.8$ & $1.42 \pm 0.06$ & $34.5 \pm 4.0$ \\
U12 & $47(7.5)$ & $11.6 \pm 0.4$ & $1.49 \pm 0.05$ & $38.9 \pm 3.7$ \\
\hline U13 & $43(6.9)$ & $13.1 \pm 0.4$ & $1.60 \pm 0.08$ & $46.3 \pm 7.1$ \\
\hline U14 & $62(9.9)$ & $14.0 \pm 0.4$ & $1.68 \pm 0.07$ & $56.7 \pm 8.4$ \\
\hline U15 & $67(10.7)$ & $15.0 \pm 0.7$ & $1.74 \pm 0.08$ & $63.6 \pm 8.5$ \\
\hline U16 & $61(9.8)$ & $16.2 \pm 0.5$ & $1.76 \pm 0.06$ & $68.2 \pm 7.4$ \\
\hline U19, U18 & $148(23.7)$ & $17.6 \pm 0.8$ & $1.79 \pm 0.07$ & $73.4 \pm 8.2$ \\
\hline
\end{tabular}

\section{Injury recording and definitions}

Injuries sustained by players were diagnosed and recorded by medical personnel at each club, in accordance with previously published guidelines [20] and sent anonymised to researchers in a standardised electronic spreadsheet. Injuries were recorded when they had occurred during soccer-related activity (training or match-play) and resulted in a player being unable to participate in training or competition for 24 hours or more following the incidence or onset of injury. A player was classified as injured until they were able to return to full training and become available for match selection, with the number of days absent calculated as the difference between the date of injury until the date of return to full training and selection availability. Categorisation of injury location and type were recorded according to previously published guidelines [20]. Severity of injury was classified according to the total number of days missed, including: minimal (1-3 days), mild (4-7 days), moderate (8-28 days) and severe (>28 days) $[14,20]$. Traumatic injury was defined as an injury with a clearly identifiable event leading to injury, whilst overuse injury was defined as an injury believed to result via gradual onset without a clear injury-inciting event. Injuries were classified as contact or non-contact depending on whether a clear incident involving contact with another player, the ball or another object was present or not. Injuries categorised as muscle rupture/strain/cramps, sprain/ligament injury or tendon injury/rupture/tendinosis/bursitis were grouped under "soft-tissue injury". Injury rate was calculated by dividing the number of injuries by the number of participating players $[4,11]$. 
Data are presented as means \pm standard deviations $(\mathrm{SD})$. The chi-square $\left(\chi^{2}\right)$ test of independence was used to compare the injury rate for the most common injury types and locations between the four nations and injury severity for each age group, while the Pearson's $\chi^{2}$ (goodness of fit) test compared the monthly distribution of injuries throughout the season for each country. Due to English and Spanish soccer seasons starting in August and the Uruguayan and Brazilian seasons beginning in February, the 10 months of the season were normalised to month number, where Month 1 represented August for England and Spain, and February for Uruguay and Brazil. All statistical analyses were performed using SPSS Version 25.0 (IBM Statistics, Chicago, Illinois) and statistical significance was set at $p<0.05$.

\section{$\underline{\text { Results }}$}

\section{Summary of injuries}

During the season, a total of 471 injuries were recorded. Twenty eight injuries were excluded because they occurred outside of soccer training or match play, leaving 443 injuries for analysis. The injury rate for all injuries in the entire cohort was 0.71 injuries per player, with 252 players from the cohort suffering at least one injury. A total of 12,143 days were lost to injury with an average of 28 (range 1 to 303) days of absence per single injury. The majority of injuries were non-contact (58.5\%) and were mainly suffered in training (54.4\%) compared to matches (40.9\%), with $4.7 \%$ from unknown soccer origin. Traumatic and overuse injuries accounted for $46.3 \%$ and $26.6 \%$ of injuries, respectively, however, $27.1 \%$ were of unspecified origin due to lack of sufficient data. Injury rates for the most recorded injuries according to chronological age group are presented in Table 2.

\section{Injury severity}

"Moderate" injuries ( 8 to 28 days, $44.2 \%$ ) represented the most frequent severity category, followed by "severe" (>28 days, 28.7\%) and "mild" (4-7 days, 18.3\%), with "minimal" injuries (1-3 days, 8.1\%) contributing fewest. There was a significant difference in the proportion of severe injuries according to chronological age group, $\chi^{2}=$ $42.19, p=0.001$ (Fig1). The U13, U14, U15, U16 and U17/U18 age groups had a significantly greater proportion of severe injuries than the U12 age group, whilst the U14 and U16 age groups also had a significantly greater proportion of severe injuries than the U19/U20/U23/Reserves age group (all $p<0.05$ ). 


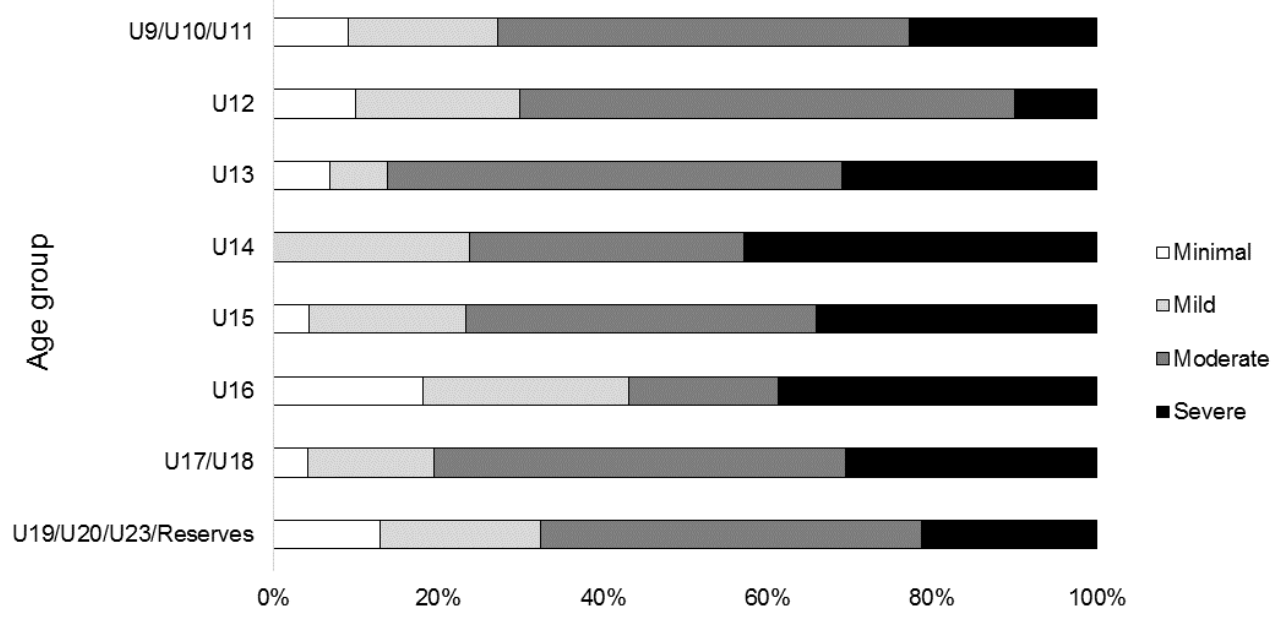

Percentage of injuries

114 Fig. 1. Distribution of injury severity according to age.

115 Table 2. Rates of most prevalent injury type and location according to chronological age group.

\begin{tabular}{|c|c|c|c|c|}
\hline & Injury Type & Rate & Injury Location & Rate \\
\hline \multirow[t]{3}{*}{ U9/U10/U11 } & Growth-related injury & 0.14 & Knee & 0.11 \\
\hline & Sprain/ligament injury & 0.06 & Foot & 0.06 \\
\hline & Other bone injury & 0.05 & Low back/sacrum/pelvis & 0.05 \\
\hline \multirow[t]{3}{*}{ U12 } & Growth-related injury & 0.30 & Low back/sacrum/pelvis & 0.33 \\
\hline & Other bone injury & 0.06 & Knee & 0.11 \\
\hline & Sprain/ligament injury & 0.04 & Ankle & 0.07 \\
\hline \multirow[t]{3}{*}{ U13 } & Growth-related injury & 0.16 & Low back/sacrum/pelvis & 0.19 \\
\hline & Sprain/ligament injury & 0.07 & Knee & 0.16 \\
\hline & Muscle rupture/strain/tear/cramps & 0.05 & Foot & 0.05 \\
\hline \multirow[t]{3}{*}{ U14 } & Muscle rupture/strain/tear/cramps & 0.15 & Ankle & 0.13 \\
\hline & Sprain/ligament injury & 0.11 & Knee & 0.11 \\
\hline & Growth-related injury & 0.08 & Low back/sacrum/pelvis & 0.10 \\
\hline \multirow[t]{3}{*}{ U15 } & Muscle rupture/strain/tear/cramps & 0.19 & Thigh & 0.16 \\
\hline & Other bone injury & 0.13 & Knee & 0.12 \\
\hline & Sprain/ligament injury & 0.09 & Low back/sacrum/pelvis & 0.07 \\
\hline \multirow[t]{3}{*}{ U16 } & Muscle rupture/strain/tear/cramps & 0.16 & Thigh & 0.20 \\
\hline & Haematoma/bruise/contusion & 0.15 & Knee & 0.11 \\
\hline & Sprain/ligament injury & 0.09 & Ankle & 0.07 \\
\hline \multirow[t]{3}{*}{ U17/U18 } & Muscle rupture/strain/tear/cramps & 0.24 & Knee & 0.17 \\
\hline & Sprain/ligament injury & 0.16 & Thigh & 0.16 \\
\hline & Haematoma/bruise/contusion & 0.05 & Ankle & 0.14 \\
\hline U19/U20/U23/ & Muscle rupture/strain/tear/cramps & 0.28 & Thigh & 0.28 \\
\hline \multirow[t]{2}{*}{ Reserves } & Sprain/ligament injury & 0.18 & Knee & 0.16 \\
\hline & Haematoma/bruise/contusion & 0.11 & Ankle & 0.11 \\
\hline
\end{tabular}


118 The most common locations were thigh, knee, ankle and low back/sacrum/pelvis (Fig 2), with the most common 119 types of injury being muscle strain/rupture/cramps and sprain/ligament injury (Fig 3). Most injuries were to the 120 lower limbs (75.3\%), and over half of all injuries were classed as soft-tissue injuries (54.0\%). Of these, muscle 121 rupture/strain/tear/cramps was most common (54.8\%), followed by sprain/ligament injury (37.7\%) and tendon injury/rupture/tendinosis/bursitis (7.5\%). Most soft-tissue injuries were non-contact (65.3\%), meaning $35.2 \%$ of all recorded injuries were non-contact soft-tissue injuries.

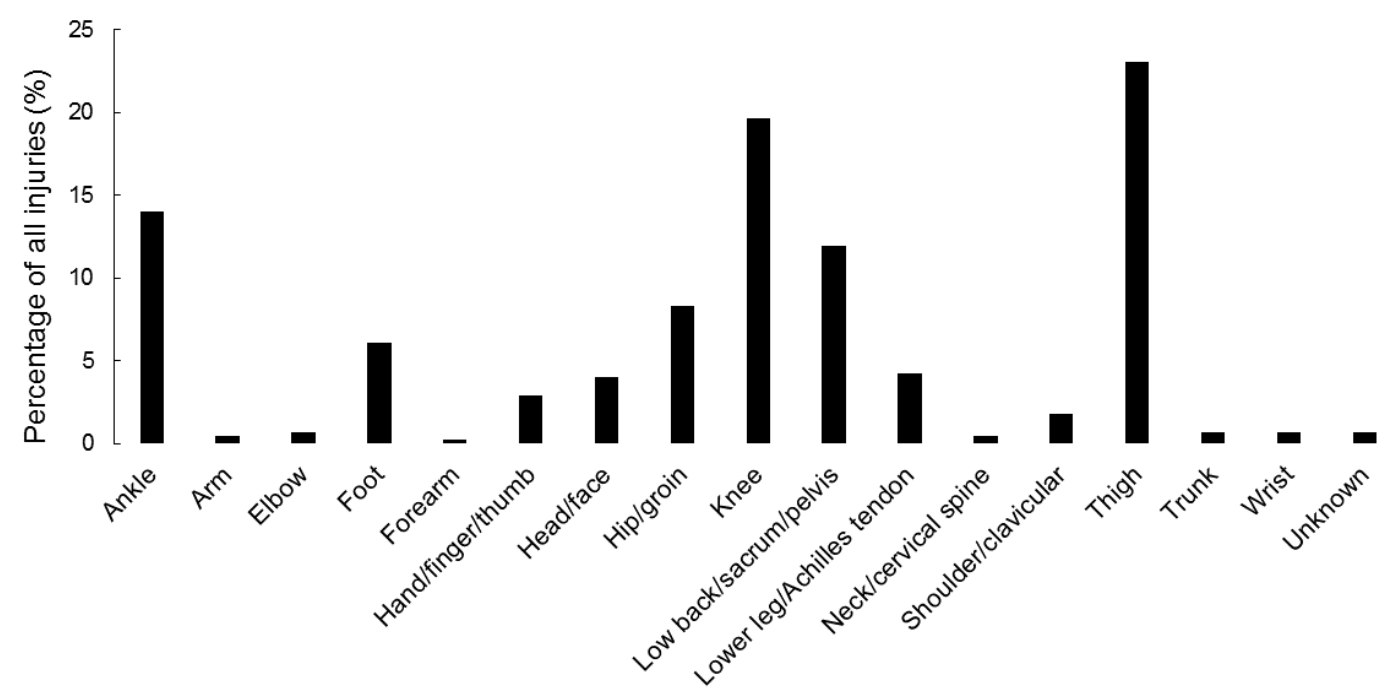

Anatomical Location

Fig. 2. Distribution of all recorded injuries based on anatomical location. 


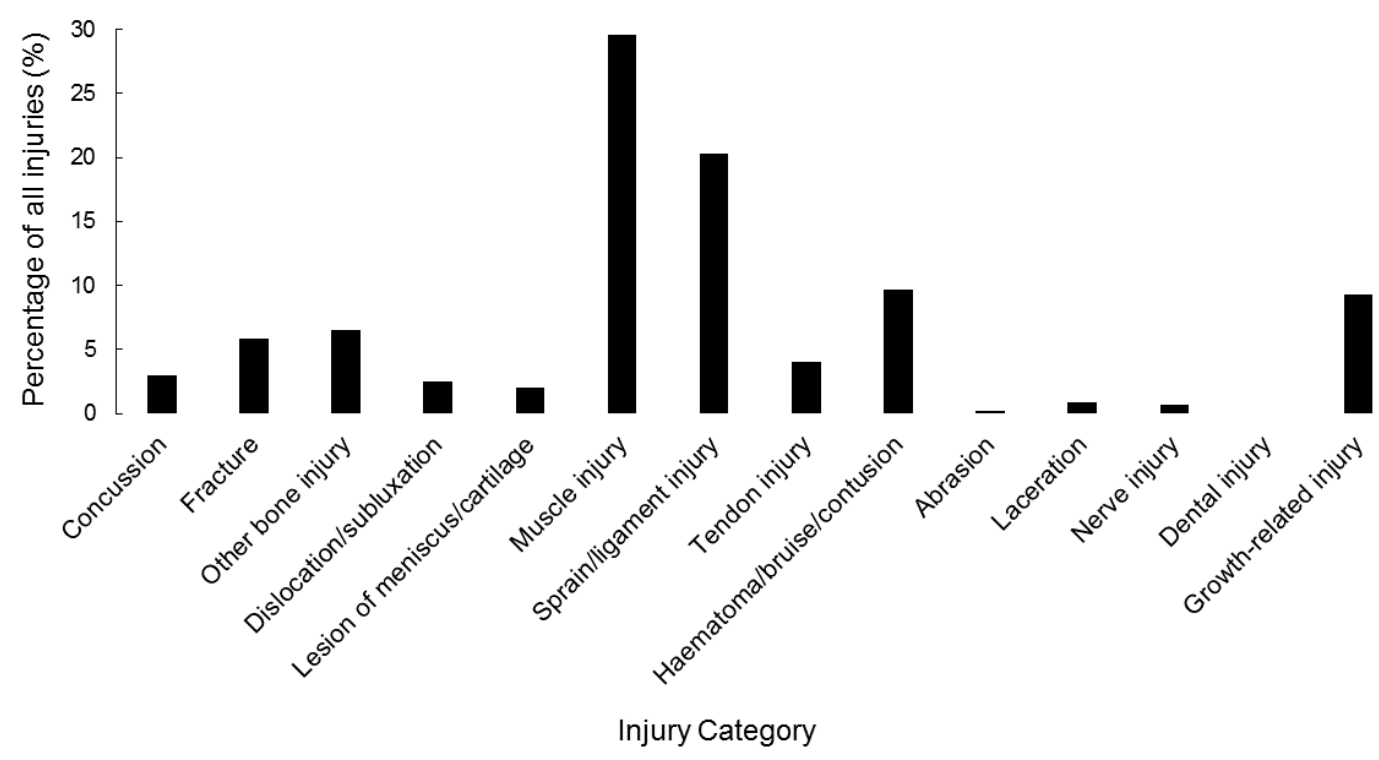

Fig. 3. Distribution of all recorded injuries based on injury type.

Muscle Injuries

There were 131 muscle/rupture/strain/tear/cramps injuries incurring 2,285 days of absence and an average of 17 (range 1 to 91 ) days lost per injury. Of all injuries in this category, $77.1 \%$ occurred through non-contact situations, and were mainly from training (58.8\%) compared to matches $(38.2 \%)$, with $3.1 \%$ of unspecified origin. Most were traumatic (39.7\%) compared to overuse $(32.8 \%)$, though $27.5 \%$ were unspecified due to lack of sufficient data. Most muscle injuries were resolved between 8 and 28 days (48.9\%), with only $17.6 \%$ requiring more than 4 weeks before return to play. The thigh was the most common site of muscle injury (59.5\%), followed by the hip/groin (19.8\%). Hamstring injuries were most frequent, accounting for 38.9\% of muscle injuries and $11.5 \%$ of all injuries.

\section{Ligament injuries}

There were 90 sprain/ligament injuries over the course of the season, with a total absence of 3,251 days and an average of 36 (range 1 to 303) days missed per injury. Half of the ligament injuries were non-contact (50.0\%) recorded during training $(54.4 \%)$ and matches $(40.0 \%)$, with $5.6 \%$ from unspecified activity. Ligament injuries were mainly traumatic $(66.7 \%)$ compared to overuse $(10.0 \%)$, with $23.3 \%$ unspecified due to lack of sufficient data. Injury severity in the sprain/ligament injury category was mainly moderate $(47.8 \%)$, followed by severe (28.9\%) and mild (18.9\%), with few minimal injuries (4.4\%). The ankle and knee were the most common sites, 
with $54.4 \%$ and $34.4 \%$ of all ligament injuries, respectively. Of the knee ligament injuries, $22.6 \%$ were to the anterior cruciate ligament, representing $42.9 \%$ of all ligament injury absence.

\section{Tendon injuries}

Tendon injury/rupture/bursitis/tendinosis represented $4.1 \%$ of all injuries, leading to 561 days of absence with a mean absence of 31 (range 6 to 117) days per injury. More than half were non-contact (55.6\%) with most during training (44.4\%) compared to matches $(33.3 \%)$, however $22.2 \%$ were during unspecified activity due to lack of sufficient data. Tendon injuries were mainly severe $(44.4 \%)$ or moderate $(38.9 \%)$, and were most common in the knee $(44.4 \%)$ and the hip/groin (27.8\%).

\section{Injury rate between countries}

Differences in injury rate were observed between countries $\left(\chi^{2}=76.61, p<0.001\right)$, with the rate of tendon injury being greater in the Brazilian cohort than the Spanish cohort $(0.06$ vs $0.01, p<0.05)$, and the rate of low back/sacrum/pelvis injury being greater in the Spanish cohort compared to the English, Uruguayan and Brazilian cohorts $(0.29$ vs $0.01,0.03$ and 0.00 , respectively, $p<0.05)$. No differences in injury rate were observed between countries for any other injury type/location (all, $p>0.05)$.

\section{Seasonal distribution of injuries}

A significant difference in the rate of injuries suffered per month of the season was observed when all countries were combined $\left(\chi^{2}=108.98, p<0.001\right)$ and when each country was analysed separately $\left(\chi^{2} \geq 91.50, p<0.001\right)$. Overall, Months 6, 2 and 10 had the highest injury rates. In English academies, Month 4 and Month 2 (November and September) had the greatest injury rates, whilst in the Spanish academy Months 6 and 7 (January and February) had equally high injury rates. For the Uruguayan academy, month 6 (July) had the highest injury rate with months 2 (March) and 10 (November) equal second. In the Brazilian academy, months 5 and 8 (June and September) shared the highest injury rates.

\section{$\underline{\text { Discussion }}$}

The primary purpose of this injury audit was to identify: (i) the most common injuries in YSP from four high-level soccer nations across two continents; (ii) which injuries caused the longest absences from training and match play; and (iii) whether any differences existed in injury rate between countries. We hypothesised that 
muscle and ligament injuries would be most prevalent and that the lower limbs would incur a considerable proportion of non-contact injuries, particularly to the thigh, knee and ankle. Our main findings confirmed these hypotheses, as well as our hypothesis that minimal differences would exist between the four nations regarding injury type and location. Importantly, these novel findings suggest that the most common types and locations of injuries in YSP do not differ between countries.

In general, the commonly recorded injury locations and types did not differ significantly between the four nations. However, we observed differences in the rates of tendon injuries and low back/sacrum/pelvis injuries. Specifically, players in our Brazilian academy had a higher rate of tendon injury compared to players in our Spanish academy, who had a higher rate of low back/sacrum/pelvis injuries compared to players from English, Uruguayan and Brazilian academies. The reasons for these differences are unclear, though we highlight the small number of tendon injuries recorded within our audit. Nevertheless, it is possible that different interpretations or diagnoses of injuries between Brazil and Spain contributed to these results. In addition, the mean age of the Spanish cohort was lower than the Brazilian cohort. We suggest that chronologically older players amongst the Brazilian cohort might influence the number of tendon injuries recorded, as they are likely to have accumulated greater soccer exposure and thus have suffered previous tendon injuries [21], although there are other possible factors that might explain the observed differences.

In attempting to explain the higher rate of low back/sacrum/pelvis injuries in Spanish players, we observed that the U12 to U15 age groups contributed more than two thirds of these injuries. This injury location comprises a broad range of possible injury types, which may be related to maladaptation of under-developed tissues/structures to loads experienced during training/match play. Interestingly, the Spanish cohort had a relatively higher number of players $(51.7 \%)$ in the U12 to U15 age groups in comparison to our English, Uruguayan and Brazilian clubs who had $31.9 \%, 34.7 \%$, and $0.0 \%$ respectively. Therefore, a greater relative number of U12 to U15 players in our Spanish cohort might have contributed to the differences observed. It is also possible that injury diagnosis and recording differs between the medical staff of different clubs or countries, based on the interpretation of injury location. Another possibility is differences in strength training practices between countries. In players performing limited strength training, these injuries could be due to low relative maximum strength or stability in players frequently required to run, jump and rotate [22]. The opposite may also occur, where players undertaking high volumes of soccer and strength training are more likely to be injured due to added stress on the lower back region. Most low back injuries in our audit occurred through overuse, as previously 
reported [22], suggesting low back/sacrum/pelvis injuries may be linked to insufficient rest and recovery.

204 Nevertheless, further research on low back/sacrum/pelvis injury in YSP is warranted.

Most injuries in our sample were non-contact, as previously reported in youth $[11,12]$ and senior players $[10,13]$ and $75.3 \%$ of injuries were in the lower limbs, supporting previous work $[4,10,15,23]$. The thigh was the most common site of injury, followed by the knee and the ankle, with muscle and ligament the most frequently injured tissues, meaning the injuries we observed were typical of a soccer population $[4,7,12,16]$. We observed hamstring muscle injuries as the single most common injury, which has been documented elsewhere $[4,13,16]$, Tendon injuries typically led to absences greater than a week, despite representing a small fraction of injuries, which is also commonly observed $[4,14,23]$. We consider this a justification for further investigation of their occurrence, particularly as injured tendons are unlikely to ever regain their pre-injured condition [24]. These data suggest further study of soft-tissue injury in high-level YSP, particularly addressing the risk factors that lead to their occurrence.

The percentage of severe injuries was greater in the U14 and U16 age groups compared to U12 and U19/U20/U23/Reserves age groups. Crucially, this would suggest that players close to the age of 14 and 16 years old miss more days per injury than other age groups. This is particularly interesting as these are the ages where biological maturation typically occurs in adolescent males, often coinciding with increments in training volume [25]. Despite YSP competing according chronological age, the timing of biological maturation is highly variable in adolescent males [17], with recent evidence demonstrating that the body composition of earlier maturing players may enhance their tolerance to increased training load [26]. Further investigation is merited to determine whether there is an association between biological maturation and injury severity, particularly between the U14 and U16 age groups, with some authors suggesting the rate and timing of skeletal maturation affect injury incidence and severity in YSP $[5,19,27]$.

Recovery from soccer injury varies considerably by the type and location of the injury, with injury severity categorised based on the number of days missed [7, 16, 20,28]. Moderate and severe injuries represented a combined $72.9 \%$ of all injuries in our audit, meaning less than $30 \%$ of injuries were resolved within a week. It is therefore abundantly clear that the significant problem caused by injury to player availability [3] extends to youth soccer. Absence periods could be influenced by coach attitudes, and whether some players are given additional time to recover compared to others who may be inadequately recovered but cleared as fit. Severe injuries represented more than a quarter of all injuries in our audit, a finding similar to some literature $[10,11,15]$ but higher than others $[13,14]$. Notably, studies with fewer severe injuries involve elite level professional (senior) 
teams, where medical assistance and facilities are likely to be superior, and players may be encouraged to return

to play quicker. Conversely, YSP may be afforded greater recovery time due to attitudes prioritising athletic development, which may supersede the desire for success. Nevertheless, a similar distribution of injury severity to that observed in our audit was evident amongst comparable cohorts [11, 15].

When collectively analysing all players, the rate of injury was dependent on the month of the season. Specifically, months 6, 2 and 10 of the playing season demonstrated the highest rate of injury. In players from English academies, month 4 and month 2 had the highest injury rates, which is in part agreement with previous literature describing an injury peak in month 2 in English academy players [11]. The same study also found another injury peak in month 6 , which is reflected in our findings that Spanish players had similarly high injury rates in months 6 and 7. In Uruguay, we observed the greatest peak in month 6 of the season, similar to the peak within our English and Spanish seasons. It is thought that higher injury rates occur in certain months following a return to activity after acute deconditioning during summer or winter break periods [11]. However, the months with the highest injury rates in Uruguayan and Brazilian academies do not follow such periods. Nevertheless, months within the second and third quarters of the season generally appear to demonstrate higher injury rates in each country, though the specific months when injuries peaked differed between countries. Not all studies report monthly differences in injury rates [29] and between-season variation has also been demonstrated [29]. We would not expect every season to be identical, thus it is not clear if the same pattern of injuries would exist amongst the same players in another season. Whilst practitioners should remain cognisant of the reasoning for elevated injury risk in periods following breaks from activity, our audit suggests this might affect some academies more than others.

We acknowledge some limitations in our injury audit. Firstly, lack of data regarding soccer activity (exposure) restricts the ability to provide accurate injury incidence data, which is typically reported per 1000 hours of soccer activity [20]. However, exposure records can lack clarity regarding the nature and intensity of activity, which also limits comparison between research studies even when it is available. Nevertheless, information regarding the training schedules and practices in each country could offer greater insight into the observed differences in our study. Secondly, nearly half of our cohort were above the U16 group, meaning much of our injury data may be more representative of post-pubertal players. Older players will have accrued greater soccer exposure since they began playing, which will increase their risk of injury [30], with older players more likely to have suffered one or more previous injuries due to the length of their career. It could be argued that including several soccer academies from different countries could introduce more variability from potentially different 
training styles, training volumes and coaching philosophies between countries. It is important to recognise that the accurate recording of exact injury diagnoses is challenging in all soccer clubs, as well as the fact there may be differences in diagnosis and reporting of injuries between different countries. However, one of the main aims of this audit was to investigate whether injury rates differed between YSP from England, Spain, Uruguay and Brazil, which has not been investigated before. Furthermore, we observed only small differences in injury rate in only two injury types/locations between countries, demonstrating that injuries were broadly equivalent in academies from these countries. Moreover, including fewer academies would limit the sample size considerably and restrict the ecological validity of findings, particularly if the data had come from a single academy, or a single country. Indeed, the majority of previous injury audits include several academies but from just one country $[4,10,11]$. We also acknowledge that training schedules and off-season periods may differ between clubs and countries and between age groups within the same clubs, which could be influential to the occurrence of injury, and that these are not described in our audit. It is also important to consider that injury risk relates to variables other than physiological factors, such opponent behaviour [8], which can be influenced by the level of competition and/or the reward associated with success [31], and that these are difficult to quantify. Finally, we did not provide information concerning the playing positions of the players in our audit, which we recognise as a risk factor for soccer injury [29]. Future studies should include this important variable in their injury risk analyses.

\section{Conclusion}

We conclude that injuries are prevalent in YSP, are most often suffered in the lower limbs, and that non-contact injuries to soft-tissue structures constitute a substantial proportion of injuries. Interestingly, we observed that players from our Spanish academy suffered more low back/sacrum/pelvis injuries than players from English, Uruguayan or Brazilian academies, which may be due to there being relatively more U14-U16 players in the Spanish cohort (the ages at which more low back/sacrum/pelvis injuries tended to occur). Apart from a higher rate of tendon injuries in players from Brazil than Spain, data were similar between countries concerning the main injury types/locations, suggesting injury risk in this population is similar between countries. Furthermore, players in the U14 and U16 age groups suffered a greater percentage of severe injuries compared to players of other age groups, suggesting that maturation status influences injury risk. Finally, specific months demonstrated peaks in injury rate, suggesting certain periods of the season when youth players may be at a higher risk of injury (e.g. off/mid-season breaks). 


\section{$\underline{\text { References }}$}

1. Van Mechelen, W., H. Hlobil, and H.C.J.S.m. Kemper, Incidence, severity, aetiology and prevention of sports injuries. 1992. 14(2): p. 82-99.

2. Fuller, C. and S. Drawer, The application of risk management in sport. Sports medicine, 2004. 34(6): p. 349-356.

3. Parry, L. and B. Drust, Is injury the major cause of elite soccer players being unavailable to train and play during the competitive season? Physical therapy in sport, 2006. 7(2): p. 58-64.

4. Price, R., et al., The Football Association medical research programme: an audit of injuries in academy youth football. British journal of sports medicine, 2004. 38(4): p. 466-471.

5. Le Gall, F., C. Carling, and T. Reilly, Biological maturity and injury in elite youth football. Scandinavian journal of medicine \& science in sports, 2007. 17(5): p. 564-572.

6. Arnason, A., et al., Physical fitness, injuries, and team performance in soccer. Medicine \& Science in Sports \& Exercise, 2004. 36(2): p. 278-285.

7. Read, P.J., et al., Neuromuscular Risk Factors for Knee and Ankle Ligament Injuries in Male Youth Soccer Players. Sports Medicine, 2016. 46(8): p. 1059-1066.

8. Brink, M.S., et al., Monitoring stress and recovery: new insights for the prevention of injuries and illnesses in elite youth soccer players. British journal of sports medicine, 2010: p. bjsports69476.

9. Faude, O., R. Rößler, and A. Junge, Football injuries in children and adolescent players: are there clues for prevention? Sports medicine, 2013. 43(9): p. 819-837.

10. Hawkins, R.D., et al., The association football medical research programme: an audit of injuries in professional football. British journal of sports medicine, 2001. 35(1): p. 43-47.

11. Read, P.J., et al., An audit of injuries in six English professional soccer academies. Journal of sports sciences, 2018. 36(13): p. 1542-1548.

12. Renshaw, A. and P.C. Goodwin, Injury incidence in a Premier League youth soccer academy using the consensus statement: a prospective cohort study. BMJ Open Sport \& Exercise Medicine, 2016. 2(1): p. e000132.

13. Ekstrand, J., M. Hägglund, and M. Waldén, Epidemiology of muscle injuries in professional football (soccer). The American journal of sports medicine, 2011. 39(6): p. 1226-1232.

14. Ekstrand, J., M. Hägglund, and M. Waldén, Injury incidence and injury patterns in professional football: the UEFA injury study. British journal of sports medicine, 2009: p. bjsports60582. 
15. Nilsson, T., A.H. Östenberg, and M. Alricsson, Injury profile among elite male youth soccer players in a Swedish first league. Journal of exercise rehabilitation, 2016. 12(2): p. 83.

16. Le Gall, F., et al., Incidence of Injuries in Elite French Youth Soccer Players A 10-Season Study. The American journal of sports medicine, 2006. 34(6): p. 928-938.

17. Malina, R.M., C. Bouchard, and O. Bar-Or, Growth, maturation, and physical activity. 2004: Human Kinetics.

18. Figueiredo, A., M. Coelho e Silva, and R. Malina, Predictors of functional capacity and skill in youth soccer players. Scandinavian Journal of Medicine \& Science in Sports, 2011. 21(3): p. 446-454.

19. Van der Sluis, A., et al., Sport injuries aligned to peak height velocity in talented pubertal soccer players. International journal of sports medicine, 2014. 35(04): p. 351-355.

20. Fuller, C.W., et al., Consensus statement on injury definitions and data collection procedures in studies of football (soccer) injuries. Scandinavian journal of medicine \& science in sports, 2006. 16(2): p. 8392.

21. Gajhede-Knudsen, M., et al., Recurrence of Achilles tendon injuries in elite male football players is more common after early return to play: an 11-year follow-up of the UEFA Champions League injury study. British journal of sports medicine, 2013: p. bjsports-2013-092271.

22. Purcell, L. and L.J.S.H. Micheli, Low back pain in young athletes. 2009. 1(3): p. 212-222.

23. Deehan, D., K. Bell, and A. McCaskie, Adolescent musculoskeletal injuries in a football academy. Bone \& Joint Journal, 2007. 89(1): p. 5-8.

24. Tozer, S. and D. Duprez, Tendon and ligament: development, repair and disease. Birth Defects Research Part C: Embryo Today: Reviews, 2005. 75(3): p. 226-236.

25. Elferink-Gemser, M.T., et al., The changing characteristics of talented soccer players-a decade of work in Groningen. 2012. 30(15): p. 1581-1591.

26. Campa, F., et al., The Role of Somatic Maturation on Bioimpedance Patterns and Body Composition in Male Elite Youth Soccer Players. Int J Environ Res Public Health, 2019. 16(23).

27. Van der Sluis, A., et al., Importance of peak height velocity timing in terms of injuries in talented soccer players. International journal of sports medicine, 2015. 36(04): p. 327-332.

28. Hawkins, R.D. and C.W. Fuller, A prospective epidemiological study of injuries in four English professional football clubs. British journal of sports medicine, 1999. 33(3): p. 196-203. 
29. Carling, C., E. Orhant, and F. LeGall, Match injuries in professional soccer: inter-seasonal variation and effects of competition type, match congestion and positional role. International journal of sports medicine, 2010. 31(04): p. 271-276.

30. Nédélec, M., et al., Recovery in Soccer. Sports Medicine, 2013. 43(1): p. 9-22.

31. Keller, C.S., F.R. Noyes, and C.R. Buncher, The medical aspects of soccer injury epidemiology. The American Journal of Sports Medicine, 1988. 16(1 suppl): p. S-105-S-112. 